641.5(=131.1)

https://doi.org/10.18485/italbg.2019.1.3

\author{
Francesca Pucci Donati* \\ University of Durham
}

\title{
L'ANALISI DEL TERMINE CARBONATA NELLE FONTI MEDIEVALI
}

\begin{abstract}
Tracce della cucina medievale si ritrovano non soltanto nei ricettari dell'epoca ma anche in altro tipo di fonti, quali gli statuti dei mestieri del cibo, i testi letterari, i trattati medici e quelli di stampo moralistico. Il termine italiano "carbonata", per esempio, indica un cibo menzionato in una pluralità di documenti tale da indurre a riflettere circa il suo impiego sulle tavole degli uomini del Medioevo. Come sappiamo da diversi studi sulla storia dell'alimentazione, le preparazioni culinarie attestate nei libri di cucina dei secoli XIV-XV erano spesso definite con espressioni del tipo "cibo da signore", "pasto da villano", "bontà per i ricchi”, "adatto ai contadini". La carbonata era preparata "more rusticano", o almeno così scrissero i beccai modenesi in una loro supplica al marchese estense nel 1441; ma anche altri testi coevi italiani ed europei suggeriscono un uso similare. La creazione di un corpus documentario tratto da fonti diverse ci permette di circoscrivere meglio sia il valore sociale di questo piatto che il contesto d'uso pratico della ricetta.
\end{abstract}

Parole chiave: carbonata, cibo, cucina, Medioevo, costumi, società.

La cucina medievale poggia sui principi della scienza antica, tant'è vero che molte delle prescrizioni tramandate dai ricettari italiani ed europei dei secoli XIII-XV si ritrovano nei trattati medico-dietetici coevi, i quali a loro volta si basano sulle teorie di Ippocrate e Galeno, padri della medicina occidentale 1 . Uno dei principi più importanti dello schema teorico antico è quello del "mangiare secondo la qualità della persona"; concetto che in età classica aveva a che fare soprattutto con la scienza medica e la fisiologia del corpo, così come essa era stata concepita a partire dal corpus ippocratico,

*francesca.pucci-donati@durham.ac.uk

${ }^{1}$ La bibliografia sulla storia della medicina occidentale è estremamente vasta. Mi limito in questa sede a fornire un riferimento classico per questa letteratura, che permette di cogliere il passaggio dalla tradizione medica antica a quella medievale: Grmek (1993). Inoltre, riguardo alla scienza dietetica fondamentale è il lavoro di Marilyn Nicoud (2007) sui regimina sanitatis tardo medievali. 
mentre nel Medioevo assume soprattutto una valenza fortemente sociale ${ }^{2}$. La scienza sembra così influenzare i costumi e la mentalità dei ceti delle élites europee, fornendo a queste ultime solide argomentazioni per giustificare le distinzioni economiche sociali e culturali a tavola. Si tratta di teorizzazioni e pratiche ampiamente studiate dagli storici europei dell'alimentazione, a cominciare dai lavori degli ultimi decenni del secolo scorso ${ }^{3}$. Inoltre, la realizzazione di edizioni critiche di alcuni dei più importanti ricettari italiani e francesi, stampate proprio a partire da quel periodo, ha permesso agli studiosi di soffermarsi in maniera più approfondita sullo studio del linguaggio del cibo ${ }^{4}$. In tale prospettiva sono stati organizzati convegni e pubblicazioni circa l'esame di singoli termini o gruppi di lemmi, di espressioni, di ricette e di formule di vario genere contenenti informazioni sulla cucina medievale. L'esame dei termini che definiscono le diverse preparazioni culinarie ha permesso di mettere in luce, in non pochi casi, il contesto d'uso di un determinato cibo/piatto, l'idea che gli uomini del tempo ne avevano, nonché il valore dietetico, sociale e culturale a esso attribuito. In questo senso l'analisi delle pietanze e della loro composizione ha di frequente fornito utili chiavi di lettura per indagare la mentalità dell'epoca.

Il principio del "mangiare secondo la qualità della persona" è dunque un principio cardine della cultura medievale e le fonti letterarie, quelle trattatistiche e documentarie sono ricche di esempi eloquenti a tal riguardo. Del pari, l'iconografia - basti pensare al genere dei calendari dei mesi dipinti nei palazzi, scolpiti sulle cattedrali o raffigurati nei codici miniati - fornisce schematizzazioni suggestive su che cosa e come mangiavano i potenti, i borghesi e i contadini nel Medioevo. Numerosi studi del settore hanno messo a fuoco e spiegato le rappresentazioni più ricorrenti, probabilmente spesso non così tanto distanti dalle pratiche concrete della vita quotidiana proprie dei diversi gruppi sociali ivi raffigurati. Per quanto riguarda le fonti scritte, troviamo sovente indicazioni del genere "è pasto da villano", "è un cibo da re", "adatto ai signori" e altre espressioni simili. Esempi del genere si riscontrano nel Libreto de tutte le cosse che se magnano, trattato in volgare del medico Michele Savonarola, attivo nella

${ }^{2}$ Montanari (1994: 104-115). Sul rapporto fra scienza medica, dietetica e cucina sono illuminanti le pagine di Jean-Louis Flandrin (1997), lo storico che per primo ha affrontato lo studio della dietetica in età medievale e moderna, unitamente a quelle di Massimo Montanari (2004: 63-70) che ha approfondito lo stretto nesso esistente fra piacere e salute.

${ }^{3}$ Sul tema dell'alimentazione e le gerarchie sociali nel Medioevo e nella prima età moderna si veda in particolare Grieco (1987), Grieco (1997) e il già citato Montanari (1994).

${ }^{4}$ Riguardo al rapporto fra lingua e cucina, si vedano gli atti del convegno tenutosi a Modena nel 2007, incentrato esclusivamente su questo tema: Robustelli \& Frosini (2009). 
seconda parte del Quattrocento presso la corte estense di Ferrara ${ }^{5}$; oppure, nel ricettario quattrocentesco di Giovanni Bockenheym, cuoco di papa Martino V a Roma ${ }^{6}$. Quest'ultimo distingue pure le preparazioni culinarie in base alle nazionalità ("pro Italicis", "pro Almanis", "pro Frisonibus et Slavis", "pro Anglis") e alla morale ("pro ruffianis et leccatricibus"). Analoghe precisazioni si ritrovano sovente in diverse fonti dell'epoca, a dimostrazione del fatto che l'idea secondo cui ciascuno dovesse mangiare in base alla categoria economica e sociale di appartenenza era estremamente diffusa e trasversalmente condivisa. Che poi tali dettami fossero rispettati o trasgrediti, e in quale misura in un senso o nell'altro, non è dato sapere dai trattati medici e dai libri di cucina. Tali fonti forniscono un insieme di teorie e pratiche rivolte a un pubblico d'élite (borghesia, signori, papi, re, principi), ma soprattutto veicolano un sistema di valori e un codice di comportamenti riguardanti l'intera società del tempo, come è stato giustamente sottolineato dagli studiosi di storia dell'alimentazione.

Ulteriori informazioni circa $\mathrm{i}$ costumi alimentari in voga nei secoli tardo medievali emergono inoltre dalle fonti documentarie che narrano di fatti avvenuti o di problemi contingenti. È il caso, per esempio, di una testimonianza curiosa - da cui ha preso avvio la riflessione proposta in queste pagine - costituita da una supplica dei beccai modenesi inviata al marchese Niccolò d'Este e datata 3 ottobre 14417. In essa i macellai del contado di Modena chiedono all'allora signore della città che venga loro concesso di allevare e tenere più di otto porci a famiglia, cosa che fino a quel momento era stata proibita dagli ufficiali del marchese stesso. Essi adducono una solida motivazione a supporto di quella richiesta: con otto porci riescono a sfamare la famiglia soltanto per metà dell'anno, oltre al fatto che spesso accolgono presso le loro case amici e forestieri che abbisognano di essere nutriti e alloggiati. Per tutti costoro, gli stessi beccai sono soliti preparare "more rusticano carnibus salatis, fatiendo carbonatas et ponendo in patella": ovvero, secondo il costume dei rustici preparano carni di porco salate, facendole arrostire in padella ${ }^{8}$. L'espressione "more rusticano" identifica una

${ }^{5}$ Per l'edizione del testo si veda Nystedt (1988).

${ }^{6}$ Come edizione del testo ho utilizzato Bonardi (1995). Per l'inquadramento di Giovanni Bockenheym alla corte del papa e del suo testo nella storia della cucina medievale si veda Laurioux (2005b: 303-332).

${ }^{7}$ Archivio Storico Comunale di Modena (d'ora innanzi: ASCMo), Camera segreta, n. 7, "Statuta Comunis Mutine spectantia ad officium iudicis super victualibus et extraordinariis deputati”, 1327-152, c. 64r. Cfr. Lucchi (1963: 13).

${ }^{8}$ ASCMo, Camera segreta, n. 7, cit., c. 64r: "quod sunt magne familie et ad eorum domos concurrunt multi forenses amici et qui quos quasi continue cibant more rusticano carnibus salatis fatiendo carbonatas et ponendo in patella”. Cfr. Lucchi (1963: 13). 
modalità di preparazione non particolarmente ricercata e legata all'utilizzo del fuoco mediato soltanto da un tegame; una modalità probabilmente rivolta a una clientela dal palato non raffinato e dai gusti non eccessivamente sofisticati; oppure, abituata a viaggiare e ad adattarsi a qualsiasi tipo di situazione. La carbonata o "carbonada" infatti indicava una preparazione culinaria a base di carne di maiale salata e fortemente arrostita sulla brace di carbone (di qui il nome della ricetta), oppure arrostita su piastra o padella di ferro 9 . Uguccione da Pisa nelle sue Derivationes riporta quale sinonimo di questo piatto il lemma di carbonella ${ }^{10}$.

Dal documento in questione si intende che doveva essere una pratica corrente presso i beccai del contado modenese quella di fare la carbonata per gli amici e soprattutto per gli avventori di passaggio. Che si trattasse in ogni caso di una consuetudine diffusa emerge da varie testimonianze dell'epoca, fra cui, per esempio, quella di Jacques Despars, medico al servizio di Carlo VII re di Francia. Costui menziona nel suo Commento al Canone di Avicenna un piatto consumato nelle taverne e nelle locande francesi denominato "charbonnée", ovvero sottili fette di porco grigliate, che di norma venivano poco e mal cotte dagli esercenti, allo scopo di suscitare nei clienti il bisogno di dissetarsi spesso e in grandi quantità ${ }^{11}$. Al di là dell'opinione di Jacques Despars, dettata soprattutto dai principi dietetici del tempo, la ricetta della carbonata risultava essere di per sé stessa particolarmente salata, come spiega nel suo Libro de arte coquinaria Maestro Martino, il primo autore di un ricettario italiano (scritto in lingua volgare nella seconda metà del XV secolo). La sua opera costituisce un superamento - per qualità e stile - di quelle della tradizione precedente ${ }^{12}$ :

Togli la carne salata che [sia] vergellata di grasso et magro insieme, et tagliala in fette, et ponile accocere ne la padella et non le lassare troppo cocere. Dapoi mittile in un piattello et gettavi sopra uno pocho di zuccharo, un pocha di cannella, et un pocho di petrosello tagliato menuto. Et similemente poi fare de summata o presutto,

${ }^{9}$ Battaglia (2007: 744). Per un inquadramento della ricetta nella cucina medievale si consulti Carnevale Schianca (2011: 121-122).

${ }^{10}$ Cecchini, E. \& Arbizzoni, G., Lanciotti, S., Nonni, G., Sassi, M. G., Tontini, A. (2004: 189).

${ }^{11}$ Jacquart (1980: 50). Il contributo di Danielle Jacquart è stato pubblicato anche sul sito internet: Persee.fr/doc/bec_0373-6237_1980_num_138_1_450189. Cfr. pure Laurioux (1989: 116).

${ }^{12}$ La letteratura su Maestro Martino e il suo ricettario è ampia. Per un approfondimento del tema si rimanda alle bibliografie dei seguenti lavori: Benporat (1996), Capatti \& Montanari (1999: 13-15), Montanari (2012: 20-22). Un inquadramento generale sulla cucina italiana si trova in Montanari (2010). 
giongendoli in scambio d'aceto del sucho d'aranci, o limoni, quel che più ti piacesse, et farratte meglio bevere (Faccioli 1992: 143) ${ }^{13}$.

Secondo il dettato di Maestro Martino, occorre dunque prendere della carne salata filettata di grasso e magro, tagliarla a fette e metterla in padella senza farla cuocere eccessivamente. Alla fase della cottura segue quella, tipicamente medievale, del condimento mediante la creazione e l'impiego di una salsa a base di ingredienti agrodolci quali lo zucchero, la cannella, e il prezzemolo tagliato fino ${ }^{14}$. Similmente - recita la ricetta - si può preparare la lombata di maiale o il prosciutto, aggiungendo, in luogo dell'aceto, del succo d'arancia o di limone, a piacere; e tutto questo susciterà inevitabilmente voglia e bisogno di bere. La ricetta presenta due versioni distinte: una, contenente l'aceto e l'altra più sofisticata, in cui la salsa è composta da un agrume (per la precisione, succo d'arancio o di limone). L'osservazione finale "et farratte meglio bevere" è stata interpretata dagli storici in maniera non dissimile da quanto il medico francese Jacques Despars aveva scritto a proposito delle osterie parigine. Come si è già accennato, la carbonata era infatti comunemente considerata una pietanza che faceva venire voglia di consumare molte bevande e perciò ritenuta l'accompagnamento ideale nelle bisbocce ${ }^{15}$. È un'interpretazione che suggerisce l'associazione fra questo cibo e lo stato di ebbrezza di chi lo consumava soprattutto nelle taverne, ma che non necessariamente ha dei nessi con espressioni linguistiche di altro genere contenenti il lemma carbonata. In proposito, basti citare il curioso modo di dire "andare il mondo in carbonata". Esso significa letteralmente: andare il mondo sottosopra, abbruciare, rovinare qualcosa o qualcuno ${ }^{16}$, a causa dell'azione immoderata delle braci infuocate.

Il termine carbonata trova anche riscontri nelle fonti letterarie trequattrocentesche italiane e francesi a testimonianza, come si diceva, della diffusione di questa preparazione culinaria in area europea. Il noto novelliere italiano Franco Sacchetti narra nel suo Trecentonovelle (novella CVIII) la vicenda di Testa da Todi prevosto dei Priori di Firenze, il quale usava bere vino ogni mattina di buon'ora. Un giorno in particolare costui decide di

${ }^{13}$ Per il presente lavoro, ho utilizzato l'edizione dei testi di cucina, ormai divenuta un classico, di Emilio Faccioli. Occorre tuttavia ricordare che nel corso dell'ultimo cinquantennio sono apparse diverse edizioni critiche o semplici trascrizioni del testo di Maestro Martino. Mi limito qui a segnalare una delle più recenti: Ballerini \& Parzen (2001).

${ }^{14}$ Circa l'importanza economica e culturale delle spezie nel Medioevo, nonché il loro uso in cucina e in dietetica, fondamentali sono i lavori di Laurioux e Flandrin. Si veda per una bibliografia essenziale sull'argomento: Laurioux (1983, 1997, 2002, 2005a), Flandrin (1997), Capatti \& Montanari (1999). Cfr. Freedman (2009).

${ }^{15}$ Redon, Sabban \& Serventi (1994: 122-123).

${ }^{16}$ Tommaseo \& Bellini (1865: 336). 
accompagnare la consueta bevuta mattutina con una fetta di carne salata: "perché 'l vino non gli facesse noia, e anco per potere bere meglio, prese una fetta di carne salata, e con uno pane sotto se n'andò alla cucina, e mettendo la detta carne su la bracia, come la si fu un poco riscaldata" (Puccini 2004: 303-304), fu chiamato dai compagni perché Guglielmo, nipote del papa e suo luogotenente, era appena arrivato e aveva manifestato desiderio di parlargli. Testa "per non perdere quella sua arrosticciana o carbonata che vogliamo dire, mettela in uno pane e cacciasela sotto e giugne in sala" (Puccini 2004: 303-304), dove era atteso. Malauguratamente per il prevosto, messer Guglielmo aveva un cane che incominciò ad andare intorno a Testa, sentendo l'odore della carbonata che il prevosto nascondeva sotto la veste. L'insistenza del cane fu tale che alla fine Testa gli gettò per disperazione la pietanza estraendola dall'abito, con grande vergogna nei confronti dei compagni e di Guglielmo. Ritorna anche in questo gustoso episodio dai toni ironici l'idea della carbonata accompagnata alla bevuta, seppure nella modalità solitaria e nascosta di un religioso. Il nesso carbonata-bere-osteria ricompare d'altronde nella poesia del XV secolo, come per esempio in quella burlesca di Burchiello, che scrive nel sonetto 301: "Se Se ci è l'Ostier, ch'io vi farò godere, / Trova una carbonata, e dai da bere" (Girotto 2013: 220). Nei sonetti parodici di Luigi Pulci la carbonata è addirittura paragonata all'anima umana: "L'anima è sol, come si vede espresso, / in un pan bianco caldo un pinocchiato,/ o una carbonata in un pan fesso" (Orvieto 1986: 431).

Si tratta di un termine diffuso anche in area francese nel pieno e tardo Medioevo, se riscontriamo, oltre alla citazione rilevata nel Commento al Canone di Avicenna di Jacques Despars, attestazioni in fonti letterarie almeno di due secoli precedenti. L'occorrenza più famosa del lemma si riscontra nella letteratura d'oïl e, più precisamente, nel Chevalier au lion di Chrétien de Troyes, poema cavalleresco della fine del XIII secolo. A un certo punto del racconto, Yvain (il chevalier au lion protagonista della storia) affronta un orco enorme chiamato Harpin della Montagna. All'inizio del combattimento l'eroe riesce a sorprendere l'avversario colpendolo in piena faccia: la spada del cavaliere affetta il viso del titano tanto che "il li abat/ de la joe une charbonee" (Poirion 1994: 441), ovvero gli taglia dalla guancia una bella fetta di carne da grigliare. È qui evidente la metafora culinaria (non l'unica nel poema ${ }^{17}$ ), che induce a pensare si trattasse di una pietanza assai comune per l'epoca e probabilmente ritenuta triviale nell'opinione dei più. Il medesimo lemma ritorna ugualmente in contesti epici, sempre con un'accentuazione di "basso" corporeo.

${ }^{17}$ Già nei versi precedenti il sangue del gigante viene paragonato a una salsa (Poirion 1994: 440). 
Questi pochi esempi mostrano come il termine afferisse soprattutto sin dal XIII secolo - nella percezione di chi allora scriveva - alla sfera della materialità e del cibo. Ancora nel Cinquecento la carbonata è menzionata con toni del tutto simili negli scritti mordaci di Pietro Aretino. Gli ambiti letterari di riferimento dunque rinviano la parola e del pari la ricetta all'universo del "basso", del "more rustico" dei villani, dei contadini, dei lavoratori attivi nelle campagne e nei villaggi, in contrapposizione al mondo "cortese" e "raffinato" della rete urbana. Tuttavia, non vi è soltanto la ben nota contrapposizione città/campagna; a essa pare aggiungersene un'altra esistente all'interno delle città, ossia quella fra gli umili e i meno abbienti da un lato, e i potenti e ricchi dall'altro ${ }^{18}$. Le osterie di Parigi cui fa riferimento il medico Jacques Despars sono un esempio emblematico in tal senso ${ }^{19}$.

Con questa breve indagine intorno al termine carbonata si è cercato di proporre qualche riflessione sulle ricorrenze dello stesso in alcuni generi letterari medievali. Il contesto d'uso della parola offerto di volta in volta dalla documentazione esaminata, unitamente agli autori e ai destinatari (presunti) dei testi, ha fornito utili informazioni circa la preparazione culinaria del piatto e i luoghi dove era prevalentemente consumato, nonché il grado di apprezzamento del medesimo nell'opinione comune e presso i medici e cuochi dell'epoca. Talvolta, infine, è emerso da qualche racconto il gusto del singolo; pensiamo per esempio al divertente episodio narrato da Sacchetti, che ha come protagonista il prevosto Testa da Todi grande bevitore e mangiatore di carbonata.

\section{BIBLIOGRAFIA}

Ballerini, L. \& Parzen, J. (a cura di). (2001). Maestro Martino, Libro de arte coquinaria (con una scelta di ricette per la tavola moderna a cura di S. Barzini). Milano: Tommasi.

Battaglia, S. (2007). Grande Dizionario della Lingua Italiana. Vol. II. Torino: UTET.

Benporat, C. (1996). Cucina italiana del Quattrocento. Firenze: Olschki. Bonardi, G. (a cura di). (1995). Giovanni Bockenheym. La cucina di papa Martino $V$. Milano: Mondadori.

Capatti, A. \& Montanari, M. (1999). La cucina italiana. Storia di una cultura. Roma-Bari: Laterza.

Carnevale Schianca, E. (2011). La cucina medievale. Lessico, storia, preparazioni. Firenze: Olschki.

${ }^{18}$ Montanari (2009: 697-705).

${ }^{19}$ Sulle osterie a Parigi nel Medioevo si veda Chatelain (1898), Champion (1912). Riguardo alle insegne e alla vita di taverna a Parigi si veda inoltre Favier (2015: 116-117). 
Cecchini, E. \& Arbizzoni, G., Lanciotti, S., Nonni, G., Sassi, M. G., Tontini, A. (a cura di). (2004). Uguccione da Pisa, Derivationes, II. Firenze: Edizioni del Galluzzo.

Champion, P. (1912). Liste de tavernes de Paris d'après les documents du XVe siècle. Bulletin de la Société de l'histoire de Paris et de l'Ile-deFrance, 259-267.

Chatelain, E. (1898). Notes sur quelques tavernes fréquentées par l'Université de Paris au XIVe et XVe siècles. Bulletin de la Société de l'histoire de Paris et de l'Ile-de-France, 87-109.

Faccioli, E. (1992). L'Arte della cucina in Italia. Torino: EInAudi.

Favier, J. (2015). Le Bourgeois de Paris au Moyen Âge. Paris: Tallandier.

Flandrin, J. L. (1997). Assaisonnement, cuisine et diététique (aux XIV, $\mathrm{XV}^{\mathrm{e}}$ et XVI ${ }^{\mathrm{e}}$ siècles). In J. L. Flandrin \& M. Montanari (éd.), Histoire de l'alimentation (pp. 491-509). Paris: Fayard.

Freedman, P. (2009). Il gusto delle spezie nel Medioevo (traduzione di Domenico Giusti). Bologna: Il Mulino.

Girotto, C. A. (a cura di). (2013). Rime del Burchiello commentate dal Doni. Pisa: Edizioni della Normale.

Grieco, A. J. (1987). Classes sociales, nourriture et imaginaire alimentaire en Italie (XIVe-XVe siècles). Paris: EHESS.

Grieco, A. J. (1997). Alimentation et classes sociales à la fin du Moyen Âge et à la Renaissance. In J. L. Flandrin \& M. Montanari (éd.), Histoire de l'alimentation (pp. 479-490). Paris: Fayard.

Grmek, D. (a cura di). (1993). Storia del pensiero medico occidentale, I. Antichità e Medioevo. Roma-Bari: Laterza.

Jacquart, D. (1980). Le regard d'un médecin sur son temps: Jacques Despars. Bibliothèque de l'ècole de chartes, 138, 35-86.

Laurioux, B. (1983). De l'usage des épices dans l'alimentation médiévale. Médiévales, 5, 15-31.

Laurioux, B. (1989). Le Moyen Âge à table. Paris: Éditions Adam Biro.

Laurioux, B. (1997). Cuisine médiévales (XIV et XVe siècles). In J. L. Flandrin \& M. Montanari (éd.), Histoire de l'alimentation (pp. 459-477). Paris: Fayard.

Laurioux, B. (2002). Manger au Moyen Age. Pratiques et discours alimentaires en Europe aux XIV et XV siècles. Paris: Hachette.

Laurioux, B. (2005a). Une histoire culinaire du Moyen Age. Paris: Champion.

Laurioux, B. (2005b). De Jean de Bockenheim à Bartolomeo Scappi cuisiner pour le pape entre le XV et le XVI siècle. In A. Jamme \& O. Poncet (éd.), Offices et papauté, XIV-XVII siècle: charges, hommes, destins (pp. 303-332). Rome: École française de Rome. 
Lucchi, G. (1963). Camera Segreta. Codici Statutari, registri ed atti costitutivi della Comunità e delle Arti. Inventario. Modena: Ed. Cooperativa Tipografi.

Montanari, M. (1994). La fame e l'abbondanza. Storia dell'alimentazione in Europa. Roma-Bari: Laterza.

Montanari, M. (2004). Il cibo come cultura. Roma-Bari: Laterza.

Montanari, M. (2009). La satira del villano fra imperialismo cittadino e integrazione culturale. In R. Mucciarelli G. Piccinni \& G. Pinto (a cura di), La costruzione del dominio cittadino sulle campagne. Italia centrosettentrionale, secoli XII-XIV (pp. 697-705). Siena: Protagon.

Montanari, M. (2010). L'identità italiana in cucina. Roma-Bari: Laterza.

Montanari, M. (2012). Gusti del Medioevo. I prodotti, la cucina, la tavola. Roma-Bari: Laterza.

Nicoud, M. (2007). Les régimes de santé au Moyen Âge: naissance et diffusion d'une écriture médicale, XIIIe-XVe siècle (2 voll.). Rome: École française de Rome.

Nystedt, J. (a cura di). (1988). Libreto de tutte le cosse che se magnano: un'opera di dietetica del sec. XV. Michele Savonarola. Stockholm: Almqvist \& Wiksell international.

Orvieto, P. (a cura di). (1986). Opere minori. Luigi Pulci. Milano: Mursia.

Poirion, D. (a cura di). (1994). Oeuvres complètes. Chretien de Troyes. Paris: Gallimard.

Puccini, D. (a cura di). (2004). Il Trecentonovelle di Franco Sacchetti. Torino: Unione tipografico-editrice torinese.

Redon, O., Sabban, F. \& Serventi, S. (a cura di). (1994). A tavola nel Medioevo con 150 ricette dalla Francia e dall'Italia. Roma-Bari: Laterza.

Robustelli, C. \& Frosini, G. (a cura di). (2009). Storia della lingua e storia della cucina. Parola e cibo: due linguaggi per la storia della società italiana (Atti del VI Convegno ASLI, Associazione per la storia della lingua italiana, Modena, 20-22 settembre 2007). Firenze: Franco Cesati.

Tommaseo, N. \& Bellini, B. (1865). Dizionario della Lingua Italiana. I. Roma-Torino-Napoli: Unione Tipografico Editrice. 


\title{
THE ANALYSIS OF THE WORD CARBONATA IN THE MEDIEVAL SOURCES
}

\begin{abstract}
Summary
Evidence of medieval cooking can be found not only in the cookbooks of the time, but also in other kinds of sources, such as statutes of food crafts, literary texts, medical books and etiquette guides. The Italian term carbonata designates a medieval dish which is quoted in just such a variety of records, and thus provides us with a picture of medieval culinary habits and attitudes. As we know from various studies of food history, many of the culinary methods presented in the cookbooks of the $14^{\text {th }}$ and $15^{\text {th }}$ centuries were often defined by expressions such as "food for lords", "meal for villains", "good food for rich people", "suitable for peasants". The carbonata was prepared more rusticano, or at least this is what the butchers of Modena wrote in a petition to the marquis of Este in 1441. Equally, other Italian and European sources from the same period offer a similar suggestion for the recipe. By drawing on different sources to build a body of literature we can delineate the social value of this food as well as his context of practical usage.
\end{abstract}

Key words: carbonata, food, cooking, Middle Ages, customs, society. 